\title{
Construction of a recombinant vaccinia virus expressing Babesia gibsoni thrombospondin-related anonymous protein and evaluation of its immunogenicity in mice
}

\author{
Nakamura, C. ${ }^{1}$, Liu, M.M. ${ }^{1}$, Goo, Y.K. ${ }^{1,2}$, Zhang, G.H. ${ }^{1}$, Jia, H.L. ${ }^{1,3}$, Kumagai, A. ${ }^{1}$, Zhang, H.S. ${ }^{1,4}$, \\ Zhou, J.L. ${ }^{1,4}$, Nishikawa, Y. ${ }^{1}$ and Xuan, X.N. ${ }^{1 *}$ \\ ${ }^{1}$ National Research Center for Protozoan Diseases, Obihiro University of Agriculture and Veterinary Medicine, \\ Obihiro, Hokkaido 080-8555, Japan \\ ${ }^{2}$ Department of Parasitology and Tropical Medicine, Kyungpook National University School of Medicine, \\ Daegu, 700-422, Republic of Korea \\ ${ }^{3}$ State Key Laboratory of Veterinary Biotechnology, Harbin Veterinary Research Institute, Chinese Academy \\ of Agricultural Sciences, Harbin 150069, China \\ ${ }^{4}$ Key Laboratory of Animal Parasitology of Ministry of Agriculture, Shanghai Veterinary Research Institute, \\ Chinese Academy of Agricultural Sciences, Shanghai 200241, China \\ *Corresponding author e-mail: gen@obihiro.ac.jp \\ Received 12 December 2019; received in revised form 7 July 2020; accepted 8 July 2020
}

\begin{abstract}
Previously, we have identified a gene encoding thrombospondin-related anonymous protein of Babesia gibsoni (BgTRAP), and have shown that the antisera raised against recombinant BgTRAP expressed in Escherichia coli inhibited the growth of parasites. In the present study, a recombinant vaccinia virus expressing the BgTRAP (VV/BgTRAP) was constructed. A specific band with a molecular mass of $80 \mathrm{kDa}$, which is similar to that of native BgTRAP on the merozoites of B. gibsoni, was detected in the supernatant of VV/ BgTRAP-infected RK13 cells. Mice inoculated with VV/BgTRAP produced a specific antiBgTRAP response. The antiserum against VV/BgTRAP showed reactivity against the native BgTRAP on parasites. These results indicated that the recombinant vaccinia virus expressing BgTRAP might be a vaccine candidate against canine $B$. gibsoni infection.
\end{abstract}

\section{INTRODUCTION}

Babesia gibsoni is a tick-borne apicomplexan parasite that causes piroplasmosis in dogs. The disease is characterized by remittent fever, progressive anemia, hemoglobinuria, and marked splenomegaly and hepatomegaly; in addition, it sometimes causes death. B. gibsoni infection is endemic in many regions of Asia, Africa, Europe, and America (Casapulla et al., 1998; Zhou et al., 2002). Recently, this disease has frequently been observed in companion animals, becoming a significant problem from a clinical point of view (Farwell et al., 1982; Adachi et al., 1993).
The development of a vaccine that would reduce or prevent the clinical symptoms of canine B. gibsoni infection is considered to be the best approach for controlling the disease. However, no vaccine is currently available. Therefore, there is a need to develop an effective vaccine to control $B$. gibsoni infection in dogs.

Thrombospondin-related anonymous proteins (TRAPs) are a conserved family identified in several apicomplexans, including Plasmodium spp., Toxoplasma gondii, Cryptosporidium parvum, Eimeria tenella, Neospora caninum, and B. bovis (Tomley et al., 1991; Trottein et al., 1995; Robson et al., 1997; Templeton et al., 1997; 
Wan et al., 1997; Spano et al., 1998; Lovett et al., 2000; Gaffar et al., 2004). Previously, we identified and characterized a $B$. gibsoni TRAP (BgTRAP) (Zhou et al., 2006). The amino acid sequence of BgTRAP consists of several typical regions, including a signal peptide, a vonWillebrand factor a domain, a thrombospondin type 1 domain, a transmembrane region, and a cytoplasmic C-terminus. The BgTRAP showed bivalent cation-independent binding to canine RBC, and the specific antiserum was found to inhibit the growth of B. gibsoni in infected severe combined immune deficiency (SCID) mice given canine RBC. These results suggest that the BgTRAP plays a critical role in the erythrocyte invasion by B. gibsoni and that it might be a logical candidate for a vaccine antigen as well as a serodiagnostic reagent (Zhou et al., 2006).

A live antigen delivery system has many advantages for the large-scale development of vaccines. It is easy to produce, resistant to environmental extremes, and less expensive than other systems. In addition, the recombinant live vaccine elicits strong host immunity against itself as well as other heterologous antigens. Recombinant vaccinia viruses have been demonstrated to be effective antigen delivery systems for infectious diseases (Panicali et al., 1983; Smith et al., 1983; Moss et al., 1984; Tsukiyama et al., 1989; Ertl \& Xiang, 1996). In this study, we constructed a recombinant vaccinia virus expressing BgTRAP and evaluated its immunogenicity against $B$. gibsoni in mice.

\section{MATERIALS AND METHODS}

\section{Cells and viruses}

The vaccinia virus $\mathrm{LC} 16 \mathrm{mO}(\mathrm{mO})$ strain and its recombinants were propagated in rabbit kidney (RK13) cells in Eagle's minimum essential medium (Sigma, USA) supplemented with $8 \%$ fetal bovine serum (FBS).
Construction of a recombinant vaccinia virus expressing the BgTRAP or green fluorescent protein (GFP)

The recombinant vaccinia virus expressing BgTRAP (VV/BgTRAP) or GFP (VV/GFP) was constructed as follows. The fragment containing the open reading frame of the BgTRAP gene was amplified from cDNAs of $B$. gibsoni by polymerase chain reaction (PCR) using a set of primers, 5'-ACGAA TTCAAGCATGGCGAGGATGAAG-3' and 5'ACGAATTCTCAGGCCCACATGGCTTCA-3' (Zhou et al., 2006). The PCR product was cloned into the EcoRI site of the cloning vector, $\mathrm{pBluescriptSK} \mathrm{(pBS)} \mathrm{(Stratagene,}$ USA). The plasmid pBS/BgTRAP was then cut with $E c o$ RI, and the fragment (2,227 bp) containing BgTRAP was blunted using Klenow Fragment (Takara, Japan) and cloned into the $S a l$ site of the vaccinia virus transfer vector, pAK8 (Yasuda et al., 1990). Plasmid pCX-EGFP was cut with EcoRI, and the fragment (732 bp) containing EGFP was blunted using the Klenow Fragment and cloned into the SalI site of pAK8. RK13 cells infected with the vaccinia virus $(\mathrm{mO})$ were transfected with the recombinant transfer vectors. Thymidine kinasenegative (TK-) viruses were isolated by a plaque assay on 143TK- cells in the presence of 5-bromo-2'-deoxyuridine at a concentration of $100 \mu \mathrm{g} / \mathrm{ml}$ (Yasuda et al., 1990).

\section{Indirect fluorescent antibody test (IFAT)}

RK13 cells infected with VV/BgTRAP or VV/GFP were placed on slides, air-dried, and then fixed with acetone for $20 \mathrm{~min}$. The diluted (appropriate dilutions were made in 10\% FBS in PBS (FBS-PBS)) anti-BgTRAPspecific rabbit serum (Zhou et al., 2006) was applied as the first antibody on fixed smears and incubated for $1 \mathrm{hr}$ at $37^{\circ} \mathrm{C}$. After three washings with PBS, Alexa-Fluor ${ }^{\circledR}$ 488conjugated goat anti-rabbit immunoglobin G (IgG) (Molecular Probes, USA) was subsequently applied (1:200 dilution in FCS-PBS) as a secondary antibody and 
incubated for another $1 \mathrm{hr}$ at $37^{\circ} \mathrm{C}$. After three washings with PBS, the glass slides were covered with a glass cover slip. The slides were examined under a fluorescent microscope.

The IFAT for determining the BgTRAP on $B$. gibsoni merozoites using the antiVV/BgTRAP antibody was carried out as described previously (Zhou et al., 2006), and the cells were observed under a confocal laser scanning microscope (TCS NT, Leica, Germany).

\section{SDS-polyacrylamide gel electrophoresis (SDS-PAGE) and Western blot analysis}

RK13 cells infected with VV/BgTRAP or VV/GFP were cultured on 6-well plates with a $0.5 \mathrm{ml}$ medium. At the end of the culture, the culture medium was harvested by centrifugation, and the cells were harvested in $1 \mathrm{ml}$ PBS. The cells were then washed three times and sonicated. The cells or culture supernatants were mixed with an equal volume of a $2 \times$ SDS gel-loading buffer under reducing conditions. The samples were boiled for $5 \mathrm{~min}$, and each $10 \mathrm{\mu l}$ of sample was then subjected to SDS-PAGE. After SDS-PAGE, the protein bands in the gel were electrically transferred to a membrane (Immobilon transfer membrane, Millipore, USA). The membrane was blocked with PBS containing $3 \%$ skim milk and then incubated with antiBgTRAP rabbit serum diluted 1:200 with PBS containing $3 \%$ skim milk at $37^{\circ} \mathrm{C}$ for $1 \mathrm{hr}$. The membrane was washed three times and incubated with HRPO-conjugated goat anti-rabbit IgG diluted 1:2,000 with PBS containing $3 \%$ skim milk at $37^{\circ} \mathrm{C}$ for $1 \mathrm{hr}$. After washing three times, the bands recognized by a specific antibody were visualized by incubation with $0.5 \mathrm{mg} / \mathrm{ml} 3$ ', 3-diaminobenzine in PBS containing 0.03\% $\mathrm{H}_{2} \mathrm{O}_{2}$.

\section{Vaccination}

Female BALB/c mice (6 weeks old) were purchased from a commercial supplier (Clea, Japan). One group of mice was inoculated intraperitoneally (i.p.) with VV/ BgTRAP. Another group of mice inoculated i.p. with VV/GFP was used as a viral control.
The doses of vaccinia viruses were $1 \times 10^{6}$ plaque-forming units (pfu) per mouse. Mice were boosted with the same inoculum 14 days after the first inoculation. Serum was collected at 1-week intervals from each mouse.

\section{Measurement of $B$. gibsoni-specific antibodies}

The BgTRAP-specific immunoglobulin level in mouse serum was measured by the enzyme-linked immunosorbent assay (ELISA). Purified GST-BgTRAP or control GST (Zhou et al., 2006) was diluted in a 50 $\mathrm{mM}$ carbonate-bicarbonate buffer $(\mathrm{pH} 9.6)$ to $2 \mu \mathrm{g} / \mathrm{ml}$, and $50 \mu \mathrm{l}$ aliquots of the diluted antigen were added to each well of a 96-well ELISA plate (Nunc, Denmark). The plate was incubated at $4^{\circ} \mathrm{C}$ overnight and washed once with PBS containing 0.05\% Tween 20 (PBS$\mathrm{T})$. The residual binding sites were then blocked with PBS containing 3\% skim milk for $1 \mathrm{hr}$ at $37^{\circ} \mathrm{C}$. Each well was washed once with PBS-T, and $100 \mu \mathrm{l}$ of serum samples diluted to 1:100 with PBS containing 3\% skim milk was added to duplicate wells for each sample. The plate was incubated at $37^{\circ} \mathrm{C}$ for $1 \mathrm{hr}$. After washing 6 times with PBS-T, the plate was incubated with HRPOconjugated goat anti-mouse IgG antibody at $37^{\circ} \mathrm{C}$ for $1 \mathrm{hr}$. After washing 6 times with PBS-T, $100 \mu$ l of substrate was added to each well and incubated at room temperature for $1 \mathrm{hr}$. The absorbance at $415 \mathrm{~nm}$ was measured by using an ELISA plate reader (Corona, Japan) and is shown as the distance between the GST-BgTRAP and control GST.

\section{RESULTS}

In order to develop an effective recombinant vaccine against $B$. gibsoni infection in dogs, a recombinant vaccinia virus expressing BgTRAP was constructed. The BgTRAP gene was inserted into the TK gene of the vaccinia virus $\mathrm{mO}$ strain under the control of the earlylate promoter for the vaccinia virus $7.5 \mathrm{kDa}$ polypeptide. To determine whether the BgTRAP was expressed in RK13 cells by the recombinant vaccinia virus, the VV/BgTRAP- 
infected cells were examined by IFAT using anti-BgTRAP mouse sera. As shown in Fig. 1, specific fluorescence was observed in VV/ BgTRAP-infected cells but not in VV/WTinfected cells.

To determine the molecular mass of the BgTRAP expressed by recombinant VV/BgTRAP, Western blot analysis was performed. A specific band with a molecular mass of $80 \mathrm{kDa}$, which was similar to that of native BgTRAP on the merozoites of $B$. gibsoni, was detected in the supernatants of VV/BgTRAP-infected RK13 cells (Fig. 2).

Figure 3 shows that a specific antibody response was obtained in mice inoculated with recombinant VV/BgTRAP but not in mice inoculated with the control virus VV/ GFP. The antibody response against BgTRAP was gradually increased after boosting the inoculation with recombinant VV/BgTRAP. The BgTRAP-specific antibody induced in mice reacted strongly with intact $B$. gibsoni merozoites, as judged by IFAT (Fig. 4). Specific fluorescence seemed to distribute on the micronemal protein of the parasites.

\section{DISCUSSION}

Vaccinia viruses have been widely used as live vectors to express foreign genes, mainly from other infectious viruses. In general, the immunization of laboratory animals or natural host animals with these recombinant vaccinia viruses could induce neutralizing antibodies and protect the animals from challenge infections with corresponding infectious viruses. Recently, vaccinia virus vector has been also used as live vector to express foreign genes from protozoan parasites, and demonstrated that the animals inoculated recombinant vaccinia vaccines could induce protective immunity against virulent parasite infections (Honda et al., 1998; Miyahara et al., 1998; Nishikawa et al., 2001).

TRAP from Plasmodium is the essential adhesion needed for sporozoite motility and liver cell invasion (Naitza et al., 1998). Moreover, recent findings, including the identification of the Plasmodium merozoite TRAP-homologue, have shown a conserved molecular motor of cell invasion and gliding

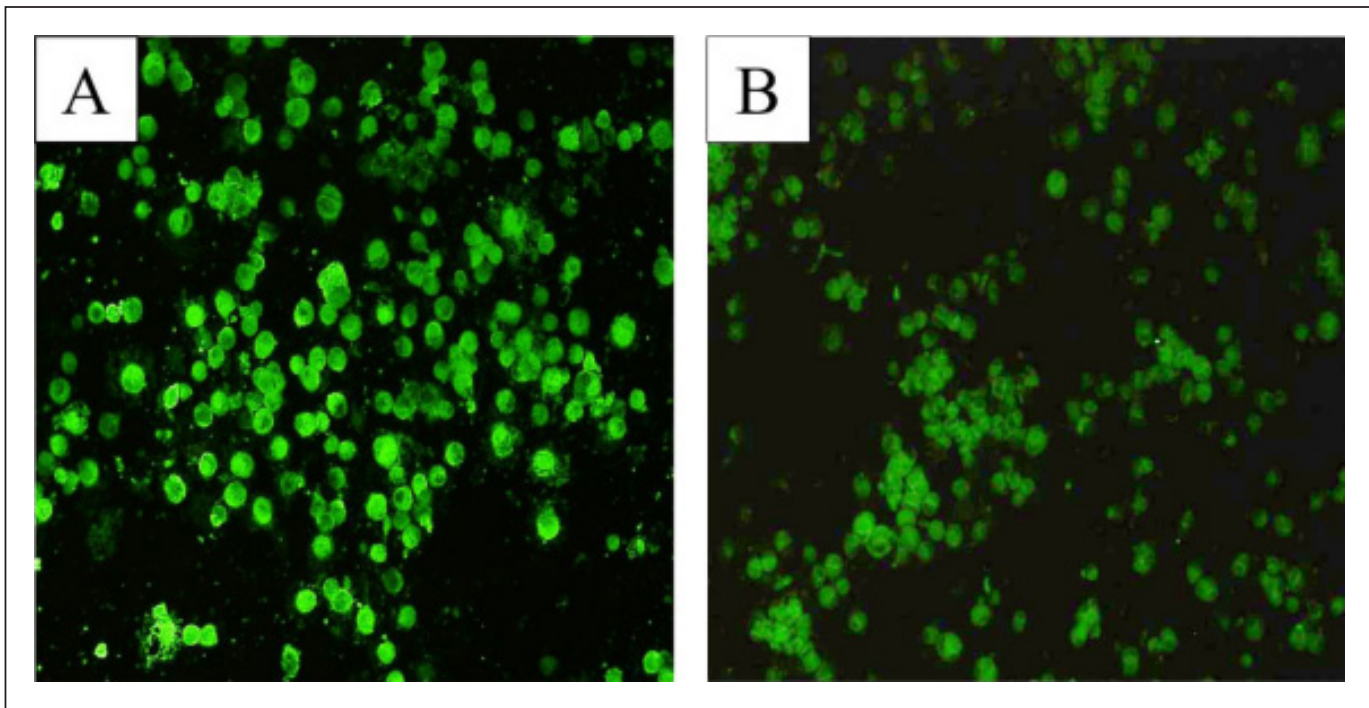

Figure 1. IFAT of recombinant BgTRAP expressed in RK13 cells by a vaccinia virus. (A) VV/BgTRAPinfected or (B) VV/wt-infect RK13 cells (48 h post infection) were reacted with anti-BgTRAP-specific rabbit serum followed by Alexa Flour-488-conjugated secondary antibodies. Alexa Flour-488-stained proteins are visualized in green. 


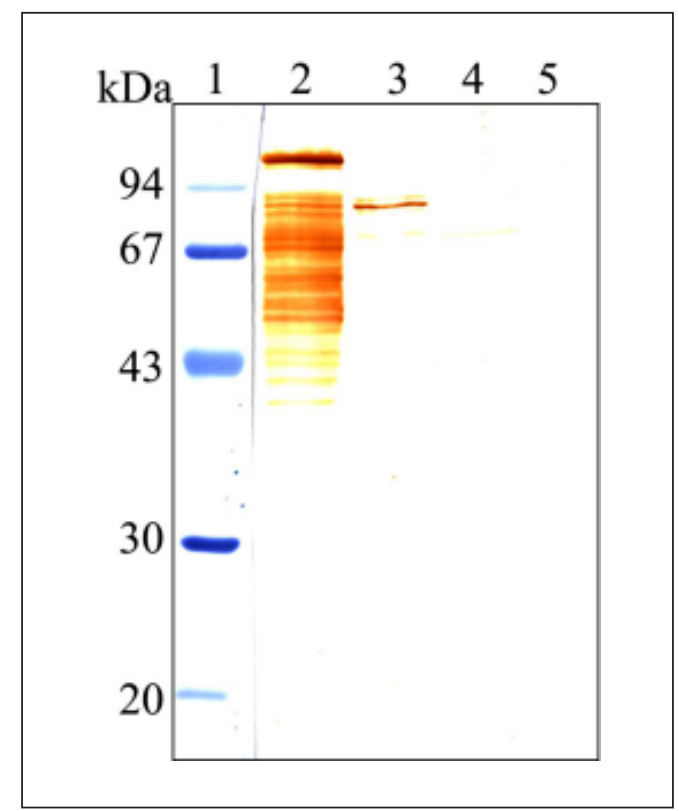

Figure 2. Western blot analysis of a recombinant vaccinia virus using anti-BgTRAP rabbit serum. Cell lysates of RK13 cells infected with VV/BgTRAP (lane 2), culture supernatants of RK13 cells infected with VV/BgTRAP (lane 3), cell lysates of RK13 cells infected with VV/GFP (lane 4), and culture supernatants of RK13 cells infected with VV/GFP (lane 5) were separated by SDS-polyacrylamide gel electrophoresis following Western blotting analysis using anti-BgTRAP rabbit serum as the primary antibody. A specific band with a molecular mass of $80 \mathrm{kDa}$, which was similar to that of native BgTRAP on merozoites of $B$. gibsoni, was detected in the supernatants of VV/BgTRAP-infected RK13 cells. Lane 1 shows molecular mass markers.

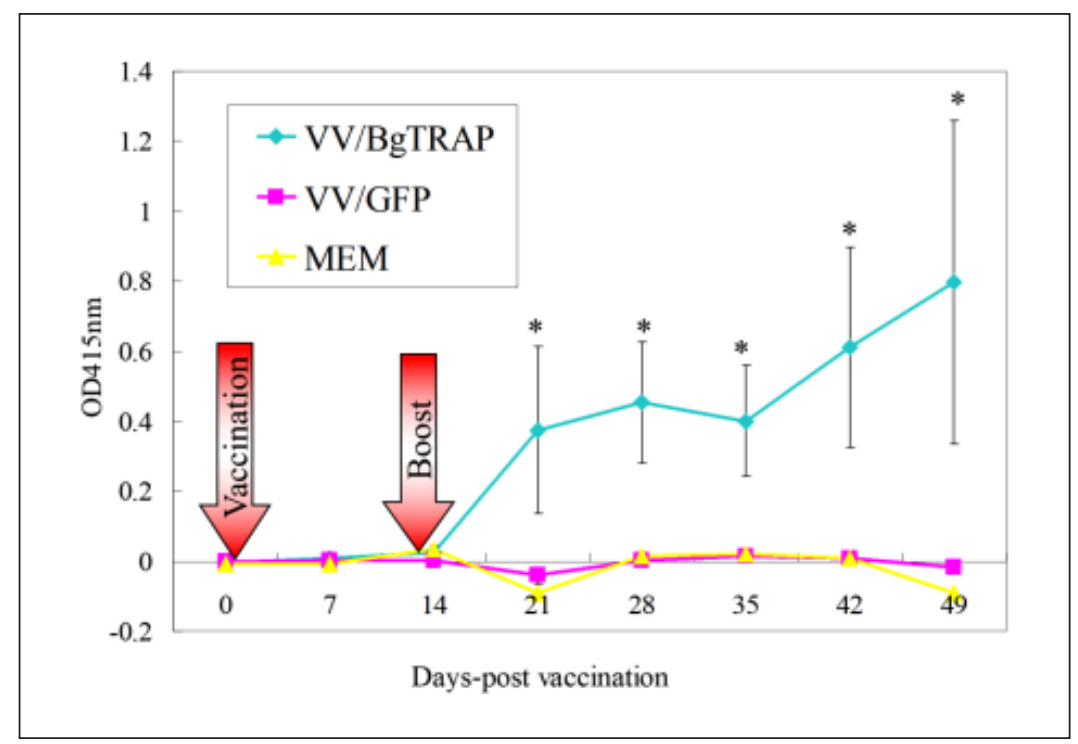

Figure 3. B. gibsoni $i$-specific antibody responses of mice vaccinated with recombinant vaccinia viruses and control mice inoculated with VV/GFP. Mice were inoculated with $1 \times 10^{6} \mathrm{pfu}$ of vaccinia viruses on days 0 and 13. Serum samples were collected at 1-week intervals from each mouse, and B. gibsonispecific antibody responses were measured by ELISA. Antibody titers were expressed as the absorbance at $415 \mathrm{~nm}$. *, The level of antibody of mice vaccinated with VV/BgTRAP was significantly higher than that of control mice $(\mathrm{P}<0.05)$. $\diamond$, VV/BgTRAP-infected; $\square$, VV/GFP-infected; $\triangle$, MEM-inoculated. 


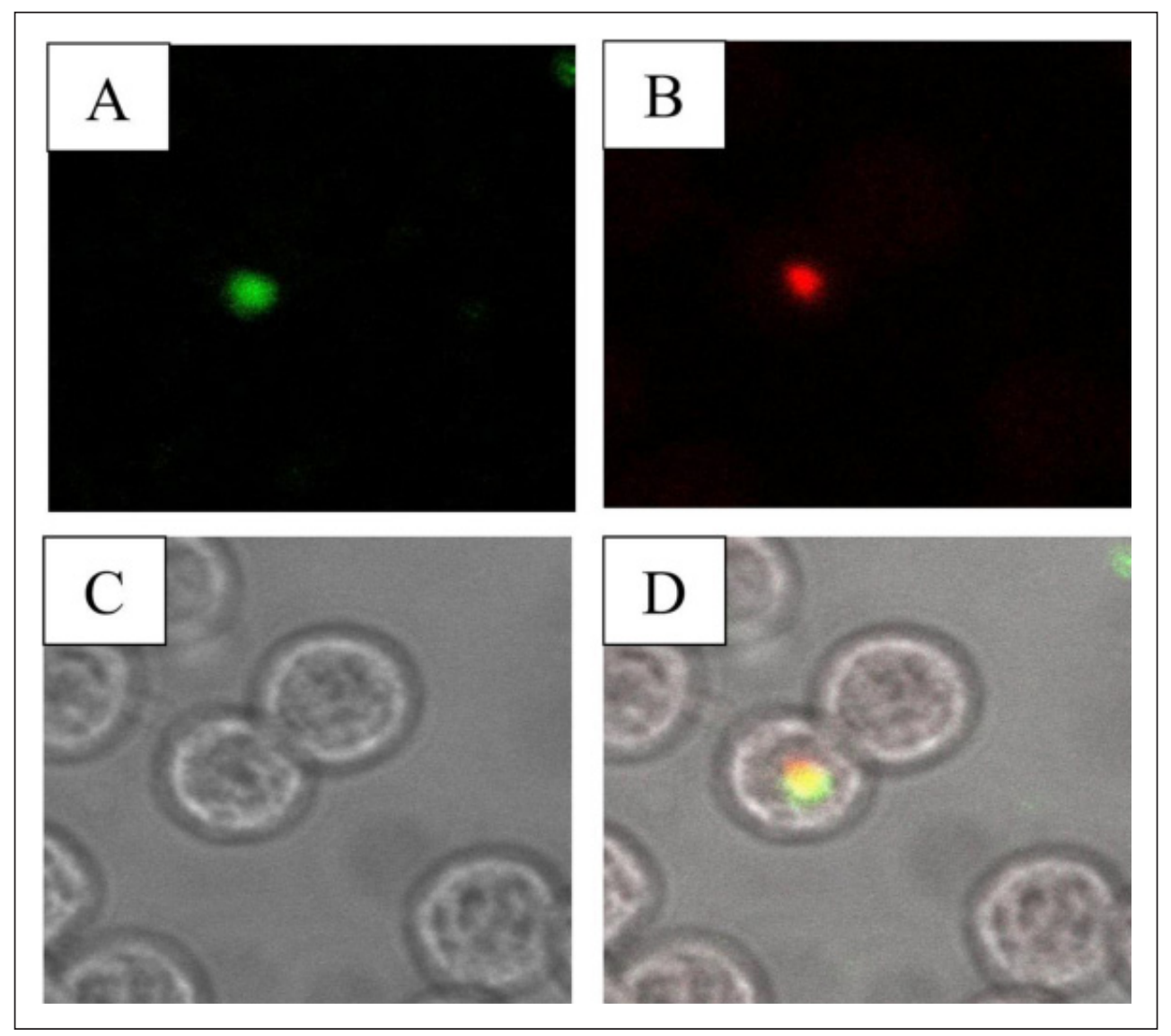

Figure 4. Observation of parasite antigen recognized by a mouse anti-VV/BgTRAP serum in confocal laser micrographs. A, Immunofluorescent staining of B. gibsoni merozoites with mouse anti-VV/BgTRAP serum. B, Propidium iodide staining of B. gibsoni merozoite nuclei. C, The phase-contrast images of B. gibsoni merozotites. D, Panels A and B overlaid on panel C. The images were derived from a single section.

motility across malaria life cycle stages and other apicomplexan parasites (Baum et al., 2006). Previously, we identified the TRAPhomologue from B. gibsoni and provided direct evidence of this protein binding to erythrocytes (Zhou et al., 2006). The antiserum against recombinant BgTRAP recognized an $80 \mathrm{kDa}$ protein in the lysate of infected erythrocytes (RBCs), which was detectable in the micronemal area of the parasite by confocal microscopic observation. The BgTRAP showed a bivalent cation-independent binding to canine RBC, and the specific antiserum was found to inhibit the growth of B. gibsoni in the infected severe combined immune deficiency mice given canine RBC (Zhou et al., 2006).

In this study, we constructed a recombinant vaccinia virus expressing BgTRAP. A specific band of $80 \mathrm{kDa}$, which was similar to that of native BgTRAP expressed in B. gibsoni, was detected in the supernatants of VV/BgTRAP-infected RK13 cells. The extra bands from the cell lysate of RK13 cells infected with VV/ BgTRAP suggest that it undergoes limited processing and that only mature BgTRAP was secreted into the supernatant. The antibody response against the BgTRAP was gradually increased after boosting the immunization with VV/BgTRAP. The antiserum against the 
recombinant BgTRAP was detectable in the micronemal area of the parasite by confocal microscopic observation. The mechanisms of immunity to babesial parasites are hypothesized to require both innate and adaptive responses that include both CD4+ $\mathrm{T}$ cells and a neutralizing antibody. Because Babesia parasites only infect erythrocytes, the adaptive immune response to subsequent infection and protection against clinical disease is dependent on the presentation of parasite antigens by antigen-presenting cells to CD4+ T lymphocytes (Brown \& Palmer, 1999; Hemmer et al., 2000; Brown, 2001). The control of Babesia infection is likely to be mediated by the destruction of infected erythrocytes by activated splenic macrophages (Brown \& Palmer, 1999; Brown, 2001) and by neutralizing antibodies directed against extracellular merozoites. Therefore, it is important to lead specific antibodies to Babesia parasites for the development of a $B$. gibsoni vaccine. Whether the antibody induced in mice by VV/BgTRAP can inhibit the $B$. gibsoni was still not confirmed in the present study due to the fact that wild type mice are not susceptible for B. gibsoni infection. However, our previous study has evidenced that the antibody produced in rabbit by $E$. coli-expressed BgTRAP can confer the protective passive immunity against $B$. gibsoni infection in SCID mice given canine RBC (Zhou et al., 2006). Therefore, the antibody produced in mice by VV/BgTRAP is speculated to having inhibitory ability against B. gibsoni parasites. Further study is needed to confirm our speculation by using dogs, the only natural host of $B$. gibsoni.

In conclusion, a recombinant vaccinia virus expressing BgTRAP was constructed, and its antigenicity in a laboratory animal was evaluated. These results indicated that the recombinant vaccinia virus expressing BgTRAP might be a vaccine candidate against canine $B$. gibsoni infection. Our next step will be immunization trails with dogs to evaluate the potency of VV/BgTRAP as a live vaccine to control the canine babesiosis caused by B. gibsoni.
Acknowledgments. This work was supported by a grant from the 21st Century COE Program (A-1) and a Grant-in-Aid for Scientific Research, both from the Ministry of Education, Culture, Sports, Science, and Technology of Japan.

\section{REFERENCES}

Adachi, K., Ueno, C. \& Makimura, S. (1993). Immunosuppression in dogs naturally infected with Babesia gibsoni. Journal of Veterinary Medical Science 55: 503505.

Baum, J., Richard, D., Healer, J., Rug, M., Krnajski, Z., Gilberger, T.W., Green, J.L., Holder, A.A. \& Cowman, A.F. (2006). A conserved molecular motor drives cell invasion and gliding motility across malaria lifecycle stages and other apicomplexan parasites. Journal of Biological Chemistry 281: 5197-5208.

Brown, W.C. (2001). Molecular approaches to elucidating innate and acquired immune responses to Babesia bovis, a protozoan parasite that causes persistent infection. Veterinary Parasitology 101: 233-248.

Brown, W.C. \& Palmer, G.H. (1999). Designing blood-stage vaccines against Babesia bovis and B. bigemina. Parasitology Today 15: 275-281.

Casapulla, R., Baldi, L., Avallone, V., Sannino, R., Pazzanese, L. \& Mizzoni, V. (1998). Canine piroplasmosis due to Babesia gibsoni clinical and morphological aspects. Veterinary Record 142: 168-169.

Ertl, H.C. \& Xiang, Z. (1996). Novel vaccine approaches. Journal of Immunology 156: 3579-3582.

Farwell, G.E., LeGrand, E.K. \& Cobb, C.C. (1982). Clinical observations on Babesia gibsoni and Babesia canis infections in dogs. Journal of the American Veterinary Medical Association 180: 507-511. 
Gaffar, F.R., Yatsuda, A.P., Franssen, F.F. \& de Vries, E. (2004). A Babesia bovis merozoite protein with a domain architecture highly similar to the thrombospondin-related anonymous protein (TRAP) present in Plasmodium sporzoites. Molecular and Biochemical Parasitology 136: 25-34.

Hemmer, R.M., Ferrick, D.A. \& Conrad, P.A. (2000). Role of T cells and cytokines in fatal and resolving experimental babesiosis: protection in TNFRp55-/mice infected with the human Babesia WA1 parasite. Journal of Parasitology 86: $736-742$.

Honda, Y., Waithaka, M., Taracha, E.L., Duchateau, L., Musoke, A.J. \& McKeever, D.J. (1998). Delivery of the Theileria parva $\mathrm{P} 67$ antigen to cattle using recombinant vaccinnia virus: IL-2 enhances protection. Vaccine 16: 12761282.

Lovett, J.L., Howe, D.K. \& Sibley, L.D. (2000). Molecular characterization of a thrombospondin-related anonymous protein homologue in Neospora caninum. Molecular and Biochemical Parasitology 107: 33-43.

Miyahara, Y., Garcia-Sastre, A., Rodriguez, D., Rodriguez, J.R., Murata, K., Tsuji, M., Palese, P., Esteban, M., Zavala, F. \& Nussenzweig, R.S. (1998). Recombinant virus expressing a human malaria antigen can elicit potentially protective immune $\mathrm{CD}^{+}$responses in mice. Proceedings of the National Academy of Sciences of the United States of America 95: 3954-3959.

Moss, B., Smith, G.L., Gerin, J.L. \& Purcell, R.H. (1984). Live recombinant vaccinia virus protects chimpanzees against hepatitis B. Nature 311: 67-69.

Naitza, S., Spano, F., Robson, K.J. \& Crisanti, A. (1998). The thrombospondin-related protein family of apicomplexan parasites: the gears of the cell invasion machinery. Parasitology Today 14: 479-484.
Nishikawa, Y., Inoue, N., Xuan, X., Nagasawa, H., Igarashi, I., Fujisaki, K., Otsuka, H. \& Mikami, T. (2001). Protective efficacy of vaccination by recombinant vaccinia virus against Neospora caninum infection. Vaccine 19: 1381-1390.

Panicali, D., Davis, S.W., Weinberg, R.L. \& Paoletti, E. (1983). Construction of live vaccines using genetically engineered pox viruses: biological activity of vaccinia virus recombinants expressing influenza virus hemagglutinin. Proceedings of the National Academy of Sciences of the United States of America 80: $5364-5368$.

Robson, K.J., Naitza, S., Barker, G., Sinden, R.E. \& Crisanti, A. (1997). Cloning and expression of the thrombospondin-related adhesive protein gene of Plasmodium berghei. Molecular and Biochemical Parasitology 84: 1-12.

Smith, G.L., Murphy, B.R. \& Moss, B. (1983). Construction and characterization of an infectious vaccinia virus recombinant that expresses the influenza virus hemagglutinin gene and induces resistance to influenza infection in hamsters. Proceedings of the National Academy of Sciences of the United States of America 80: 7155-7159.

Spano, F., Putignani, L., Naitza, S., Puri, C., Wright, S. \& Crisanti, A. (1998). Molecular cloning and expression analysis of a Cryptosporidium parvum gene encoding a new member of the thrombospondin family. Molecular and Biochemical Parasitology 92: 147-162.

Templeton, T.J. \& Kaslow, D.C. (1997). Cloning and cross-species comparison of the thrombospondin-related anonymous protein (TRAP) gene from Plasmodium knowlesi, Plasmodium vivax, and Plasmodium gallinaceum. Molecular and Biochemical Parasitology 84: 13-24. 
Tomley, F.M., Clarke, L.E., Kawazoe, U., Dijkema, R. \& Kok, J.J. (1991). Sequence of the gene encoding an immunodominant microneme protein of Eimeria tenella. Molecular and Biochemical Parasitology 49: 277-288.

Trottein, F., Triglia, T. \& Cowman, A.F. (1995). Molecular cloning of a gene from Plasmodium falciparum that codes for a protein sharing motifs found in adhesive molecules from mammals and Plasmodia. Molecular and Biochemical Parasitology 74: 129-141.

Tsukiyama, K., Yoshikawa, Y., Kamata, H., Imaoka, K., Asano, K., Funahashi, S., Maruyama, T., Shida, H., Sugimoto, M. \& Yamanouchi, K. (1989). Development of heat-stable recombinant rinderpest vaccine. Archives of Virology 107: 225235.

Wan, K.L., Carruthers, V.B., Sibley L.D. \& Ajioka, J.W. (1997). Molecular characterization of an expressed sequence tag locus of Toxoplasma gondii encoding the micronemal protein MIC2. Molecular and Biochemical Parasitology 84: 203-214.
Yasuda, A., Kimura-Kuroda, J., Ogimoto, M., Iyamoto, M., Sata, T., Sato, T., Takamura, C., Kurata, T., Kojima, A. \& Yasui, K. (1990). Induction of protective immunity in animals vaccinated with recombinant vaccinia viruses that express preM and E glycoproteins of Japanese encephalitis virus. Journal of Virology 64: 2788-2795.

Zhou, J., Mulenga, A., Yamasaki, M., Ohashi, K., Maede, Y. \& Onuma, M. (2002). Babesia gibsoni: molecular cloning and characterization of Rab6 and Rab11 homologues. Experimental Parasitology 101: 210-214.

Zhou, J., Fukumoto, S., Jia, H., Yokoyama, N., Zhang, G., Fuzisaki, K., Lin, J. \& Xuan, X. (2006). Characterization of the Babesia gibsoni P18 as ahomologue of thrombospondin related adhesive protein. Molecular and Biochemical Parasitology 148: 190-198. 